\title{
O estágio extracurricular na formação profissional: a opinião dos estudantes de fisioterapia
}

\author{
The academic training for the professional education: the opinion of the \\ Physiotherapy students
}

Ramon Távora Viana1, Glaucus Moura Moreira', Luana Torres Monteiro Melo', Nathalia Parente De Sousa1, Ana Cristhina De Oliveira Brasil2 ${ }^{2}$ Ana Paula Vasconcellos Abdon ${ }^{2}$

RESUMO I Investigar a opinião dos estudantes de Fisioterapia em relação ao estágio extracurricular e a influência dessa prática para sua formação profissional. Realizou-se um estudo transversal com alunos de Fisioterapia de uma instituição de ensino superior do estado do Ceará, Brasil. A amostra foi composta de 157 estudantes, sendo 29 homens (18,5\%) e 128 mulheres (81,5\%), com média de idade 22,6士4,02 anos, do segundo ao último semestre da graduação. Aplicou-se um questionário com 20 questões objetivas abordando a vivência e repercussões dessa prática. Do total de alunos pesquisados, 61 (38,9\%) afirmaram ter participado de estágio extracurricular. Desses, 55 (90,2\%) foram motivados pela necessidade de adquirir experiência; 53 (86,9\%) realizaram intervenções nos pacientes; e 13 (21,3\%) com carga horária de 15 a 20 horas/semanais. Porém, 36 acadêmicos (59,0\%) o fizeram em desacordo com as normas do Conselho Federal de Fisioterapia e Terapia Ocupacional. Os acadêmicos consideraram que o estágio extracurricular foi uma ferramenta de aprimoramento para sua formação profissional, pois propiciou o exercício de procedimentos e técnicas mais comuns da atuação do fisioterapeuta. Entretanto, alerta-se para a necessidade de fiscalização dos órgãos competentes, para que o aluno exerça funções compatíveis com o seu nível de qualificação.

Descritores I estágio clínico; estudantes; Fisioterapia; educação superior.

\begin{abstract}
I This study aimed to investigate the opinion of the Physiotherapy students on the extracurricular internship and the influence of this practice on their professional formation. A cross-sectional study was performed with Physical Therapy students from a higher education institution in Ceará, Brazil. The sample consisted of 157 students, 29 men (18.5\%) and 128 women (81.5\%), with mean age of $22.6 \pm 4.02$ years, from second to last semester of graduation. A questionnaire was applied with 20 objective questions covering the experiences and repercussions of this practice. A total of 61 (38.9\%) surveyed students affirmed to have participated of extracurricular internship. Out of these, 55 (90.2\%) were motivated by the necessity of acquiring experience; 53 (86.9\%) were performing therapeutic interventions in patients; and 13 (21.3\%) had 15 to 20 working hours. However, 36 academics (59.0\%) did work in disagreement with the rules of the Federal Council of Physical Therapy and Occupational Therapy. The students considered the extracurricular internship as a tool of improvement of their professional formation, because it allowed the practice of common physical therapist daily life activities procedures and techniques. There is a necessity for competent organizations to inspect these kind of activities, so that the students can practice tasks accordingly their qualification level.
\end{abstract}

Keywords I clinical clerkship; students; Physical Therapy Specialty; education, higher. 


\section{INTRODUÇÃO}

De acordo com as diretrizes curriculares dos cursos de graduação em Fisioterapia, é necessário habilitar profissionais críticos e reflexivos, aptos a atuar em diferentes cenários de prática, em equipes multidisciplinares e capazes de atender às demandas da sociedade. Isso implica transformações educacionais que assegurem uma formação generalista de profissionais fisioterapeutas, aptos a atuar em todos os níveis de atenção à saúde, através de ações de prevenção, promoção, proteção e recuperação da saúde de forma individual e coletiva ${ }^{1}$.

A carga horária mínima do curso deverá apresentar 4.000 horas, na qual uma parte desta destina-se ao estágio curricular sob supervisão do docente, equilibrado entre as diferentes áreas de atuação, níveis de atenção e recursos terapêuticos ${ }^{2}$. A universidade tem como uma das principais preocupações a adequação da matriz curricular, para que os alunos possam iniciar as práticas de estágio o mais precocemente possível, já que durante a graduação, o acadêmico de Fisioterapia tem a necessidade de vivenciar o estágio para adquirir habilidades e atitudes mais consistentes ${ }^{3}$.

O estágio curricular com supervisão do docente deve estimular a relação ensino-aprendizagem, complementando a formação do educando com o treinamento prático em situação real, que ao mesmo tempo é educativa, formativa e presta serviços à comunidade ${ }^{4}$. O objetivo é aperfeiçoar o acadêmico em sua totalidade, respeitando o embasamento ético e disciplinar da profissão, procurando desenvolver indivíduos críticos e agentes de transformação social ${ }^{5}$.

Apesar dessas preocupações por parte das instituições de ensino superior, muitos alunos ainda buscam estágios fora da proposta curricular, fato que ocorre em várias profissões da área da saúde. Essa atitude pode ocasionar prejuízo à formação acadêmica desses alunos, caso alguns pontos negativos sejam observados, como: i) ausência de vínculos com instituições de ensino; ii) falta de preparo dos profissionais para acompanhar e educar as ações do estagiário; iii) insegurança do aluno; iv) inadequação dos ambientes de estágio com o nível de conhecimento e de habilidade do estagiário; dentre outros, acarretando um possível aprendizado falho e que não atinja os objetivos propostos.

Acredita-se que estudos com esse enfoque sejam importantes para buscar dados sobre os estágios extracurriculares e as repercussões dessa prática no aprendizado dos acadêmicos, evitando o confronto com uma realidade danosa para ele e para os pacientes e a errônea substituição do fisioterapeuta pelo estagiário ${ }^{6}$.
Este estudo objetivou investigar a opinião dos estudantes de Fisioterapia em relação ao estágio extracurricular e a influência dessa prática para sua formação profissional.

\section{MÉTODOS}

Realizou-se uma pesquisa do tipo transversal e survey, em uma instituição de ensino superior (IES), no estado do Ceará, Brasil, no período de Fevereiro a Setembro de 2010.

A população de estudo foi composta por acadêmicos do curso de Fisioterapia de uma IES privada do estado do Ceará, independente do sexo e idade. Foi definido como critério de inclusão estar regularmente matriculado na graduação em Fisioterapia no primeiro semestre de 2010 e concordar em participar da pesquisa, assinando o termo de consentimento livre e esclarecido.

O curso de fisioterapia da IES em estudo possui um total de 4.500 horas, sendo 2.016 horas de disciplinas teóricas, 144 horas para disciplinas optativas e 2.340 horas de disciplinas práticas. Dessas, 648 horas para prática assistida e 900 horas para estágio supervisionado. Os estágios supervisionados em saúde coletiva são realizados em unidades básicas de atenção primária à saúde, nas quais existe o Programa de Saúde da Família; o ambulatorial, em clínicas e hospitais com assistência secundária e o hospitalar, em hospitais de atenção terciária e alta complexidade, todos vinculados e com convênio com a universidade.

A amostra foi calculada baseando-se no número de alunos por semestre (do segundo ao nono), sendo representada por $7 \%$ dos alunos no mesmo semestre. Levando em consideração o intervalo de confiança de $95 \%$, foi calculada a proporção através do software OpenEpi versão 2.3.1 (disponível on-line), que resultou no total de 101 participantes. Entretanto, para evitar que alunos de algum semestre não participassem da amostra, foi definida a igualdade do número de alunos de cada semestre.

Após o cálculo das proporções dos alunos por semestre, estabeleceu-se um número fixo de 20 alunos de cada semestre a partir do segundo ao nono, totalizando 160 alunos. Três participantes foram excluídos da pesquisa por não responderem a todos os itens do questionário.

A seleção ocorreu de maneira informal nos ambientes do campus da IES, como centro acadêmico, centro de convivência, hall dos blocos do curso e biblioteca. Após o consentimento do acadêmico, aplicou-se o questionário pelos pesquisadores previamente treinados. 
O questionário continha 20 perguntas objetivas abordando faixa etária, gênero, situação acadêmica, vivência do estágio extracurricular, área de atuação, carga horária e repercussões dessa prática. Esse questionário foi elaborado pelos pesquisadores baseado no estudo de Taquette, Costa-Macêdo e Alvarenga ${ }^{7}$.

Os dados foram analisados através da estatística descritiva e inferencial pelo programa Statistical Package for the Social Sciences (SPSS) versão 17.0, com nível de significância de 5\% ( $\mathrm{p} \leq 0,05)$.

Para analisar associação entre as variáveis aplicou-se o teste do $\chi^{2}$ através da divisão da amostra em dois grupos: o primeiro, formado por alunos que cursavam do segundo ao quinto semestre e o segundo, pelos que cursavam do sexto ao nono semestre. A divisão dos semestres utilizada foi feita seguindo as normas do estágio intervencionista para estudantes de Fisioterapia, que o permite a partir do sexto semestre (Resolução no 139/99 COFFITO) ${ }^{8}$.

O presente estudo foi aprovado pelo Comitê de Ética em Pesquisa da Universidade de Fortaleza (COÉTICA), com o Parecer de no 375/08, em obediência à Resolução no 196/96 do Conselho Nacional de Saúde/Ministério da Saúde9.

\section{RESULTADOS}

A amostra caracterizou-se por 29 homens (18,5\%) e

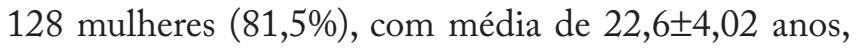
variando entre 18 a 41 anos de idade.

Em relação à participação em estágio extracurricular, $61(38,9 \%)$ acadêmicos disseram já ter participado ou estarem atualmente desenvolvendo essa atividade. Desses, 53 (86,9\%) informaram que foi de caráter intervencionista, $13(21,3 \%)$ relataram a carga horária de 15 a 20 horas semanais de trabalho, $16(26,2 \%)$ na frequência de 5 vezes/semanais e $34(55,7 \%)$ permaneceram no estágio entre 1 a 6 meses (Tabela 1 ).

Sobre a área de atuação, detectou-se que a traumatologia e a ortopedia foram relatadas por 40 acadêmicos (65,6\%), a hidroterapia por 17 (27,9\%), seguida pela fisioterapia cardiorrespiratória e dermatofuncional, ambas por dois alunos (3,3\%). Nenhum aluno citou a área da neurologia. Os recursos fisioterapêuticos mais utilizados foram a cinesioterapia e a eletrotermofototerapia, ambas citadas por $46(31,7 \%)$ dos pesquisados. A terapia manual foi citada por $35(24,1 \%)$ e, por último, a mecanoterapia por $18(12,4 \%)$.
Em relação ao motivo que levou os alunos a procurar aprendizado complementar ao da graduação, 55 $(90,2 \%)$ apontaram para a necessidade de adquirir experiência, 4 (6,6\%) motivados pela remuneração, 1 (1,6\%) apontou a curiosidade e 1 acadêmico (1,6\%) a influência de família e amigos.

Ao analisar a associação entre as características e repercussões do estágio com o semestre letivo, detectou-se que 44 acadêmicos $(72,2 \%)$ estavam entre o segundo ao quinto semestre. Desses, 36 (59,0\%) desempenhavam atividades intervencionistas nos pacientes; 23 (37,7\%) estavam satisfeitos (entre muito a extremamente) com o estágio; $28(45,9 \%)$ vivenciaram a avaliação dos seus pacientes; $39(63,9 \%)$ adquiriram novos conhecimentos; 37 (60,6\%) recomendavam o estágio; 35 (57,3\%) não relataram prejuízo na vida acadêmica com a realização do estágio; e 21 (34,4\%) recebiam remuneração (Tabela 2).

Tabela 1. Distribuição dos acadêmicos segundo o tempo dedicado ao estágio extracurricular

$\begin{array}{llrr}\text { Parâmetros } & & \mathbf{n} & \% \\ \text { Horas semanais de estágio } & \text { 2 a } 4 \mathrm{~h} & 13 & 21,3 \\ & 4 \text { a } 8 \mathrm{~h} & 12 & 19,7 \\ & 8 \text { a } 12 \mathrm{~h} & 13 & 21,3 \\ & 12 \text { a } 15 \mathrm{~h} & 10 & 16,4 \\ & 15 \text { a } 20 \mathrm{~h} & 13 & 21,3 \\ \text { Frequência por semana } & \text { 1 vez/semana } & 5 & 8,2 \\ & \text { 2 vezes/semana } & 16 & 26,2 \\ & \text { 3 vezes/semana } & 14 & 23,0 \\ & 4 \text { vezes/semana } & 10 & 16,4 \\ & 5 \text { vezes/semana } & 16 & 26,2 \\ \text { Permanência no estágio } & \text { 1 a } 6 \text { meses } & 34 & 55,7 \\ & \text { 7 a 12 meses } & 7 & 11,5 \\ & \text { mais de } 12 \text { meses } & 6 & 9,8 \\ & \text { em andamento } & 9 & 14,8 \\ & \text { outros } & 5 & 8,2\end{array}$

Tabela 2. Análise da associação entre as características e repercussões do estágio extracurricular com o semestre letivo dos acadêmicos de Fisioterapia

\begin{tabular}{llccc} 
Parâmetros & & $\begin{array}{c}\text { Abaixo do } 5^{\circ} \\
\text { semestre }\end{array}$ & $\begin{array}{c}\text { Acima do } \\
6^{\circ} \text { semestre }\end{array}$ & Valor p \\
Tipo de estágio & Intervenção & 36 & 17 & 0,05 \\
& Observação & 8 & 0 & \\
\multirow{4}{*}{ Satisfação pessoal } & Muito pouco & 3 & 1 & 0,87 \\
& Pouco & 4 & 3 & \\
& Razoável & 14 & 5 & \\
& Muito & 17 & 5 & \\
Vivência da avaliação & Extremamente & 6 & 3 & \\
\multirow{4}{*}{ Aquisição de } & Não & 28 & 7 & 0,11 \\
conhecimentos & Sim & 16 & 10 & \\
Recomenda o estágio & Sim & 39 & 14 & 0,51 \\
\multirow{4}{*}{ Prejuízos na vida } & Não & 5 & 3 & \\
acadêmica & Sim & 37 & 14 & 0,86 \\
Remuneração & Não & 7 & 3 & \\
& Sim & 9 & 0 & 0,04 \\
& Não & 21 & 17 & \\
& & 23 & 5 & 0,19 \\
& & & 12 &
\end{tabular}


Além disso, dos 35 (57,3\%) acadêmicos que relataram participar da avaliação fisioterapêutica, somente 1 $(1,65 \%)$ não relatou ter adquirido novos conhecimentos. Dos 26 (42,6\%) que recebiam remuneração, somente 3 $(4,9 \%)$ não recomendavam o estágio (Tabela 3$)$.

\section{DISCUSSÃO}

A necessidade de adquirir experiência na área de atuação através do estágio extracurricular referida por 38,9\% dos alunos da presente pesquisa representa a busca pelo novo, mostrando o interesse por uma formação complementar prática, além da proposta pelas universidades.

O exercício profissional do estudante de Fisioterapia ocorre efetivamente a partir do sexto semestre, podendo levá-lo a procurar estágios extracurriculares fora da IES desde o início do curso, com o objetivo de iniciar o mais precocemente possível a prática profissiona ${ }^{10}$. Isso ocorre mesmo com a dedicação exclusiva que o turno integral de estudo exige e com a fiscalização do órgão responsável, o Conselho Regional de Fisioterapia e Terapia Ocupacional (CREFITO).

A busca pela aquisição de conhecimentos e habilidades práticas que vão além da proposta curricular oficial das universidades é conhecida pelo termo "currículo paralelo". Esse currículo paralelo é observado em inúmeras áreas profissionais. Ele está ligado à necessidade de se adequar à realidade do mercado de trabalho, considerado o conjunto de atividades extracurriculares desenvolvidas pelos acadêmicos com o objetivo de adquirir experiências práticas, no acompanhamento de serviços e/ou profissionais ${ }^{3,7}$.

O papel das universidades nesse processo é o de orientar e educar seus alunos quanto à importância da escolha de um estágio extracurricular adequado às leis vigentes, a procura por campos conveniados à IES e que as atividades exercidas estejam em consonância com as suas competências.

Tabela 3. Análise da associação entre as opiniões dos acadêmicos sobre o estágio extracurricular

\begin{tabular}{|c|c|c|c|c|}
\hline & & \multicolumn{2}{|c|}{ Vivência da avaliação } & \multirow{2}{*}{ Valor $p$} \\
\hline & & Sim & Não & \\
\hline \multirow{4}{*}{ Aquisição de conhecimentos } & Sim & 34 & 19 & \multirow[t]{2}{*}{0,006} \\
\hline & Não & 1 & 7 & \\
\hline & & \multicolumn{2}{|c|}{ Remuneração } & \multirow{2}{*}{ Valor $\mathrm{p}$} \\
\hline & & Sim & Não & \\
\hline \multirow[t]{2}{*}{ Recomenda o estágio } & Sim & 23 & 28 & \multirow[t]{2}{*}{0,37} \\
\hline & Não & 3 & 7 & \\
\hline
\end{tabular}

Outro ponto relevante é a responsabilidade do profissional fisioterapeuta que acompanha esse estagiário, preparando-o para atender às necessidades terapêuticas da população, assim como prever as demandas clínicas e prevenções futuras ${ }^{11}$. Entretanto, alguns alunos podem não receber essa supervisão nos estágios extracurriculares e deter uma autonomia incompatível com seu nível de formação. Além disso, essas atividades sem adequada supervisão não deveriam ser utilizadas pelas empresas e clínicas ${ }^{3,12}$.

Por meio do Conselho Federal de Fisioterapia e Terapia Ocupacional (COFFITO), o estágio supervisionado é regulado a partir do sexto semestre, sendo obrigatório o vínculo com a instituição de ensino superior $^{8}$ (Resolução no 139/99 do COFFITO). Somado a isso, no artigo $3^{\circ}$ do IV capítulo da Lei no 6.316/75 do COFFITO, o estágio deve ser acompanhado por profissionais qualificados no intuito de que o acadêmico tenha o melhor aproveitamento ${ }^{13}$. Esses documentos reforçam a preocupação com o respaldo legal do aluno, além do aprendizado.

Além da legislação da categoria profissional, o estágio passou a ser regulamentado pela Lei no $11.788^{14}$, fornecendo direitos e deveres para o estagiário tanto quanto para a concedente do estágio.

$\mathrm{Na}$ presente pesquisa detectou-se que as leis e resoluções vigentes não foram respeitadas, pois 59,0\% dos alunos que estavam nos estágios extracurriculares exerciam intervenções fisioterapêuticas incompatíveis com os seus semestres letivos (entre o segundo ao quinto semestre). Além disso, os 61 (38,9\%) que participaram do estágio fora da faculdade não relataram se o local do estágio teria vínculo com a IES, o que não é considerado uma forma de estágio regulamentado.

A preocupação dos Conselhos Federal e Regionais de Fisioterapia e das instituições de ensino pode ser observada em outras profissões da saúde. Um estudo realizado em Ribeirão Preto ${ }^{15}$ identificou as fases de aprendizagem prática dos alunos de enfermagem. $\mathrm{Na}$ primeira fase, existe a dificuldade em realizar os procedimentos práticos associada ao sentimento de ansiedade. $\mathrm{Na}$ segunda, os estudantes tentam identificar as necessidades do paciente de forma eficiente. Por fim, na terceira fase o estagiário busca equilibrar sua relação em contrapartida à sua ansiedade. Isso é de vital importância para o crescimento e adoção de uma postura profissional e ética ${ }^{16,17}$.

$\mathrm{Na}$ presente pesquisa, detectou-se que um número significativo dos estagiários (42,6\%) não participava da avaliação funcional dos pacientes por eles atendidos. Esses dados 
geram inquietações e questionamentos sobre a qualidade das atividades desenvolvidas nos estágios extracurriculares e de possíveis prejuízos na formação dos acadêmicos.

Isso pode ocasionar problemas na formação dos alunos e nas condições do atendimento aos pacientes pois, em parte, não incorporam nas suas ações as competências do profissional, como a avaliação, o diagnóstico funcional, a realização das técnicas fisioterápicas, recuperação, reavaliação e alta. Essas etapas fazem parte de um atendimento completo e efetivo do fisioterapeuta e devem ser vivenciadas pelos acadêmicos ${ }^{3,18,19}$.

Paralelo a isso, os estudantes de Fisioterapia reconhecem o ganho com a prática clínica extracurricular, pois os dados mostravam que estavam satisfeitos, adquiriam novos conhecimentos e recomendavam essa atividade.

Tal opinião está em concordância com os achados de um estudo realizado nos Emirados Árabes, no qual apontou que os alunos definem quatro categorias de crescimento durante a prática clínica: i) comportamento profissional; ii) consciência do aprendizado desenvolvido; iii) autodesenvolvimento no foco terapeuta/paciente; e iv) identificação de problemas éticos. As dificuldades encontradas foram a não aceitação do estagiário pelos pacientes, problemas éticos com os profissionais, falta de recursos e grande quantidade de pacientes diariamente. Mesmo diante das dificuldades, os estudantes referiram crescimento com a prática. Os estudantes dos Emirados Árabes foram comparados com os alunos do Canadá, onde foram encontradas as mesmas dificuldades ${ }^{20}$.

Por fim, apesar de os acadêmicos apontarem para os benefícios dos estágios extracurriculares, faz-se imprescindível a regularização e fiscalização das condições e dos campos de estágio. Isso evitaria que o estudante chegue ao campo de estágio sem a formação básica, protegendo a si e aos pacientes de erros e condutas antiéticas e técnicas inadequadas, além da exploração da mão de obra dos estagiários, que, ocupando o lugar de fisioterapeutas, estariam contribuindo para os problemas de caráter trabalhista da profissão $0^{8,12,21}$.

\section{CONCLUSÃO}

Os acadêmicos consideraram que o estágio extracurricular foi uma ferramenta de aprimoramento para sua formação profissional, pois propiciou exercício de procedimentos e técnicas mais comuns da atuação do fisioterapeuta, reforçando o aprendizado propiciado pela graduação.
Entretanto, alerta-se para a necessidade de fiscalização dos CREFITOS e do questionamento da responsabilidade das empresas ou clínicas que oferecem o estágio, uma vez que irregularidades legais foram observadas. Destaca-se, ainda, que o aluno deve exercer funções compatíveis com o seu nível de qualificação durante sua formação como profissional de saúde.

\section{REFERÊNCIAS}

1. Brasil. Ministério da Educação. Resolução CNE/CES no 4, 1902/2002. Institui diretrizes curriculares nacionais do curso de graduação em Fisioterapia. Conselho Nacional de Educação, Brasília, 2002.

2. Brasil. Ministério da Educação. Parecer CNE/CES no 213/2008. Dispõe sobre carga horária mínima e procedimentos relativos à integralização e duração dos cursos graduação em Biomedicina, Ciências Biológicas, Educação Física, Enfermagem, Farmácia, Fisioterapia, Fonoaudiologia, Nutrição e Terapia Ocupacional, bacharelados, na modalidade presencial. Conselho Nacional de Educação/Câmara de Educação Superior, Brasília, 2008.

3. Vieira PS, Baggio A, Maraschin R. Estudo de Fisioterapia e Implicações para o Exercício Profissional. Saúde Rev. 2007;9(21):41-47.

4. Rodrigues MSP, Leitão GCM. Estágio Curricular Supervisionado com ênfase no desenvolvimento da autonomia e da responsabilidade. Texto contexto-enferm. 2000;9(3):216-229

5. Coury HJCG, Vilella L. Perfil do pesquisador fisioterapeuta brasileiro. Rev. bras. fisioter. 2009;13(4):356-63.

6. Jette DU, Bertoni A, Coots R, Johnson H, McLaughlin C, Weisbach C. Clinical instructors' perceptions of behaviors that comprise entrylevel clinical performance in physical therapist students: a qualitative study. Phys Ther. 2007;87(7):833-43.

7. Taquete SR, Costa-Macedo LM, Alvarenga FBF. Currículo paralelo: uma realidade na formação dos estudantes de medicina da UERJ. Rev. bras. educ. med. 2003:27(3):171-6.

8. COFFITO. Resolução no 139/92 - alterada pela Resolução no 153/1993. Dispõe sobre as atribuições do Exercício da Responsabilidade Técnica nos campos assistenciais da Fisioterapia e da Terapia Ocupacional e dá outras providências. COFFITO. 1992;28;139.

9. Brasil. Resolução CNS n.․ 196, de 10 de outubro de 1996. Aprova diretrizes e normas regulamentadoras de pesquisa envolvendo seres humanos. Diário Oficial da União. 1996 out; Seção 1(201):21082.

10. Werneck MAF, Senna MIB, Drumond MM, Lucas SD. Nem tudo é estágio: contribuições para o debate. Ciênc saúde colet. 2010;15(1):221-31

11. Domingues RCL, Amaral E, Zeferino AMB. Os diferentes olhares na avaliação de alunos em estágio clínico supervisionado. Rev Ass Med Bras. 2009;55(4):458-62.

12. Renner AF, Goldim JR, Prati FM. Dilemas éticos presentes na prática do fisioterapeuta. Rev bras fisioter. 2002;6(3):135-8.

13. COFFITO. Resolução no 6.316 de 17 de dezembro de 1975 que dispõe sobre a criação e regulamentação do Conselho Federal e os Conselhos Regionais de Fisioterapia e Terapia Ocupacional e dá outras providências. COFFITO. 1975 dez 17;6.316. 
14. Brasil. Ministério da Educação. Resolução CNE/CES n011.788. Dispõe sobre o estágio de estudantes; altera a redação do art. 428 da Consolidação das Leis do Trabalho - CLT, aprovada pelo DecretoLei n 5.452, de $1^{\circ}$ de maio de 1943. Conselho Nacional de Educação/ Câmara de Educação Superior. 2008.

15. Sadala MLA. Estudo da ansiedade como variável no relacionamento aluno-paciente. Rev Latino-Am Enfermagem. 1994;2(2):21-35.

16. Henriksen A, Ringsted C. Learning from patients: students' perceptions of patient-instructors. Med Educ. 2011;45(9):913-9.

17. Almeida $\mathrm{AH}$, Soares $\mathrm{CB}$. Educação em saúde: análise do ensino na graduação em enfermagem. Rev Latino-Am Enfermagem. 2011;19(3):[08 telas].
18. Maciel RV, Silva PTG, Sampaio RF, Drummond AF. Teoria, prática e realidade social: uma perspectiva integrada para o ensino de fisioterapia. Fisioter mov. 2005;18(1):11-17.

19. Silva ACL, Neves R da F, Riberto M. A formação fisioterapêutica no campo da ortopedia: uma visão crítica sob a óptica da funcionalidade. Acta fisiátrica. 2008;15(1):18-23.

20. Larin H, Wessel J, Al-Shamlan A. Reflections of physiotherapy students in the United Arab Emirates during their clinical placements: a qualitative study. BMC med educ. 2005;5(3):1-9.

21. Colliselli L, Tombini LHT, Leba ME, Reibnitz KS. Estágio curricular supervisionado: diversificando cenários e fortalecendo a interação ensino-serviço. Rev Bras Enferm. 2009;62(6):932-7. 